\section{EUROPEAN \\ POLYGRAPH}

PUBLISHED SEMI-ANNUALLY

2021 VOLUME 15 NUMBER $1(53)$

DOI: 10.2478/EP-2021-0001

\title{
Evidence Based Practice Integration into Polygraph Practice: A suggested paradigm
}

\author{
Tuvya T. Amsel \\ Amsel Psychophysiological Testing Ltd.; ta@amsel.co.il \\ Avital Ginton \\ AV-GN - Polygraph, Applications \& Research and part time teaching in Tel-Aviv University, \\ Faculty of Law (C/O Prof. Shlomo Shoam); ginton@zahav.net.il
}

Key words: Evidence-Based Practice; Polygraph; Comparison Question Test; CQT; Detection of Deception; Adaptive Polygraphy

\begin{abstract}
The philosophy of evidence-based practice advocates professionals to rely on scientific evidence. Although the idea seems obvious, misuse of the philosophy raised controversy, which created confusion and misunderstanding of the concept. Yet, since it was introduced in the nineties to the medical community and despite the controversy, it gained more and more disciples and was

\footnotetext{
${ }^{1}$ A similar but not identical article was recently published in the internal Magazine of the American Polygraph Association and permission was granted to republish it subject to mentioning their publication. 
embraced by nonmedical practitioners, including the polygraph profession. In the last decades, the polygraph community has gradually abandoned the intuitive-based polygraph practice that relies on less scientifically rooted subjective procedures and advanced toward evidence-based polygraph practice. This paper describes the evidence-based practice in general and details the practical aspects of evidence-based polygraph practice in particular, along with discussing the limitations of the current scientific research. It questions the current bone-tone trend to implement an extreme Evidence-Based approach into the polygraph practice, suggesting the practitioner to avoid a rigid "one size fit all" standardized protocols which are advocated as a must on the way to earn scientific recognition, whereas, in fact, it is the unfortunate outcome of lack of differential research data. As in the medical field, in-where the Evidence-Based practice managed to incorporate the clinical experience of experts with the hard research evidence and has not disregarded their valuable knowledge and experience, the present article calls for adopting this integrative approach in the polygraph field too and adjust the protocols to the specific circumstances of the case and the examinee in a "tailor-made" mode, which is based on existing data and flexible thinking wherever there is no data to rely on, as was suggested under the concept of "Adaptive-Polygraphy" (Ginton, 2013).

For many decades the use and accuracy of the polygraph are being questioned and criticized by jurists and academics (mostly psychologists) who emphasized the lack of standardization and claim that the Comparison Question Test (CQT) - the most commonly used polygraph method has no scientific merit. These prolonged claims (OTA, 1983; Ben-Shakhar, 2002; National Research Council, 2003) forced the profession to a defensive mode but also had a positive effect on it. Polygraph professionals and researchers funneled their efforts toward standardization and various validity studies. As part of these efforts, the industry embraced the trendy expression of evidence-based practice. An expression that was first introduced by the medical community and later spread to many other fields. Being based on scientific research, the term carries an aura of scientific precision. But what is really evidence-based practice? Is its' supportive research flawless? Does the evidence-based practice applicable to the polygraph practice? Does the claimed evidence-based polygraph practice is an authentic and genuine evidence-based practice? To answer these questions, one should start at the birthplace of the evidence-based practice: medicine.

In medical diagnosis, the physician is required to determine which disease explains the patient's symptoms. The information required for diagnosis is collected from the patient's complaint, past medical history, and physical examination. Many of the reported symptoms are non-specifically attributed to a single disease, thus requiring further inquiry by using differential diagnosis protocols, a process wherein 
a doctor differentiates between two or more conditions behind a person's symptoms (Langlois 2002). Additional laboratory and/or imagining examinations assist the physician in refining the diagnosis (Committee on Diagnostic Error 2015). A medical misdiagnosis that results in inappropriate treatment is quite a common event. According to the World Health Organization technical series (Diagnostic Errors, 2016), human factors such as distractions, interruptions, and failure in organizing the existing information by separating reliable from unreliable data, contribute to over half of the misdiagnosis cases. Youngstorm et al. (2015) blame overflow of information as a significant factor and claim that:

"Perhaps less than $0.25 \%$ of the research in most healthcare areas will combine scientific validity and clinical relevance. Who has the time to skim 400 articles to find one gem, which may or may not is helpful for the clients we will see this week?" (p. 1).

In order to "de-emphasizes intuition, unsystematic clinical experience, and pathophysiologic rationale as sufficient grounds for clinical decision making," Guyatt et al. (1992) suggested a new paradigm to teach medical practice:

"Evidence-based medicine requires new skills of the physician, including efficient literature searching and the application of formal rules of evidence evaluating the clinical literature." (p. 2420).

In 1996, Sackett et al., introduced a decision-making model of medical diagnosis named "evidence-based practice". The model combined three different elements:

1. Individual clinical expertise, i.e., the proficiency and judgment that individual clinicians acquire through clinical experience and clinical practice.

2. Patients' predicaments, rights, and preferences in making clinical decisions about their care.

3. Best available external clinically relevant research (Sackett et al., 1996, p. 71).

Shortly, according to Straus et al. (2011), Evidence-Based Practice developed as a philosophy and a set of skills to help manage information overload so that clinicians can continue to update practices with information to improve clients' care.

It should be emphasized that Sackett et al. (1996) explicitly emphasized that:

"Evidence-based medicine is not "cookbook" medicine because it requires a bottom-up approach that integrates the best external evidence with individual clinical expertise and patients' choice. It cannot result in slavish, cookbook approaches to individual patient care. 
External clinical evidence can inform but can never replace individual clinical expertise, and it is this expertise that decides whether the external evidence applies to the individual patient at all and, if so, how it should be integrated into a clinical decision. Similarly, any external guideline must be integrated with individual clinical expertise in deciding whether and how it matches the patient's clinical state, predicament, and preferences, and thus whether it should be applied" (p. 72). The emphasis on not a "cookbook" model arises from the authors fear that: "... evidence-based medicine will be hijacked by purchasers and managers to cut the costs of health care ... (which) would not only be a misuse of evidence-based medicine ..." (ibid, p. 72).

The authors fear was justified because soon enough, health care services, insurance companies, medical management, and alike harnessed the evidence-based practice to their own advantage, creating a "cookbook recipe" type evidence-based instructions and processes, which differs conceptually from the original definition that relies on the practitioner discretion rather than on a rigid instructional protocol. The general public could not distinguish between these two so different concepts, and the confusion got to the point that Division 12 (The Society of Clinical Psychology) of the American Psychological Association stopped using the term "evidence-based practice" in 2006, one year after the mother organization - the American Psychological Association - adopted a policy statement on evidence-based practice in psychology ${ }^{2}$, remarking these guidelines were not intended to support the dictation of specific forms of treatment (Webb, 2001).

The concept of a practice rooted in science was not limited to the medical community, and according to Leach (2006), the movement towards evidence-based practices has spread around to other fields, encouraging professionals and other decision-makers to pay more attention to the evidence in their decision-making. Evidence-based practice aims to eliminate unsound or outdated practices in favor of more effective ones by shifting the basis for decision-making from tradition, intuition, and unsystematic experience to firmly grounded scientific research. Today the evidence-based practice is implemented in many different areas: design of buildings and physical environment, education and teaching, laws formulation by legislators, management and decision making, law enforcement public policy, nursing, clinical psychology, social work, toxicology, and many others ${ }^{3}$.

\footnotetext{
${ }^{2}$ https://www.apa.org/practice/guidelines/evidence-based-statement

${ }^{3}$ On Evidence-Based Practice series go to: https://en.wikipedia.org/wiki/Evidence-based_practice
} 


\section{On scientific research - some aspects to consider}

Research results and conclusions are the core of "evidence-based practice", which in return calls for a broader overview of the scientific research. Science is in a constant endeavor to explore and chart unknown territories, i.e., new knowledge utilizing objective tools and research methods. Scientific research has an aura and image of being mathematical, objective, accurate, exact, methodical, precise, etc. ${ }^{4}$. However, when relying on scientific research, one should consider the following: Scientific research is a generic term for various types of research representing an inner hierarchy of validity strength. From the lowest and least evident type of expert opinion thru case reports, case-control studies, cohort studies, randomized control trials (blind and double-blind) all the way to the research type positioned on top of the hierarchy, the type that provides the most substantial and most robust evidence: the systematic review ${ }^{5}$. In addition, amongst the many published research and studies, some suffer from unrepresentative or insufficient sample size, inappropriate or no control group, misinterpreted results leading to unsupported conclusions, etc.

Last but definitely not least, due to the complexity and the multifactorial issues dealt by the life and behavioral sciences when using quantitative research approach, it is customary to use methods that target the central tendencies of a phenomenon, formalized in general principles and rules that concern most of the existing variance while sometimes treating the individual differences or the variation between existing situations, as irrelevant noise. When it comes to applications, some standards are developed and implemented to ensure that the applications are conducted within the framework posed by those rules, which is a must to avoiding chaos. However, because the standards are based on central tendencies and the variance around them, they are inefficient or even harmful to people or situations that are off the center (Ginton, 2013).

These scientific research aspects require practitioners, who rely on research-based evidence, to scrutinize any research cautiously, engaging critical thinking and abandoning the "carved in stone" approach to scientific research. Furthermore, recent years added a new gigantic concern: doubts in the scientific research's reliability and accuracy in what is referred to as the replication crisis in the social sciences and medicine. (Fanelli, D., 2009; Pashler and Wagenmakers, 2012).

\footnotetext{
${ }^{4}$ https://www.thesaurus.com/browse/scientific, access on January 12.2021 at 11:54

${ }^{5}$ University of Exter, Searching for scientific information: Medical Sciences: 3. Types of evidence Retrieved November 14, 2020, from https://libguides.exeter.ac.uk/csc2014/evidencetypes
} 
The last decades have witnessed a sharp growth in scientific publications, including papers and books, datasets, and websites (Bornmann \& Mutz, 2015; Larsen \& von Ins, 2010). Two major factors have contributed to this dynamic. In the technology sphere, the unprecedented progress of the capabilities to store and transfer information, and in the social sphere, the increasing pressure in the academic world and related institutions to publish research and other scientific work in order to keep holding the position and succeed in the desired career, a situation known as the Publish or Perish ${ }^{6}$ threat.

A major principle of the scientific method is reproducibility (repetition of experiments by independent researchers) or repeatability (repetition of experiments by the same researchers). Results obtained by an experiment and/or observational study should be achieved again with a high degree of agreement when the study is replicated with the same methodology by different researchers. Only after one or several such successful replications should a result be recognized as scientific knowledge (National Academies of Science, 2019). The massive growth of scientific publications became the breeding ground of the reproducibility crisis and the replicability crisis (Pashler \& Wagenmakers, 2012; Peng, R., 2015), i.e., Difficulties to reproduce or replicate the research, especially in social sciences and medicine.

Baker (2016) found that: "More than 70\% of researchers have tried and failed to reproduce another scientist's experiments, and more than half have failed to reproduce their own experiments. Those are some of the telling figures that emerged from Nature's survey of 1,576 researchers who took a brief online questionnaire on reproducibility in research." (pp. 452-3). The Open Science Collaboration (2015) claims that:

“... replications of 100 experimental and correlational studies published in three psychology journals using high-powered designs and original materials when available. Replication effects were half the magnitude of original effects ... representing a substantial decline... $97 \%$ of original studies had statistically significant results. $36 \%$ of replications had statistically significant results." (p. 943)

\footnotetext{
6 "Publish or perish" is an aphorism describing the pressure to publish academic work in order to succeed in an academic career. Such institutional pressure is generally strongest at research universities. Some researchers have identified the publish or perish environment as a contributing factor to the replication crisis. https://en.wikipedia.org/wiki/Publish_or_perish. Access on January 27, 2021 at $15: 15$.
} 
The growth of publications, along with the poor reproducibility and replicability rate, led two respectful scientists to claim that most published research findings are false. Prof. Ioannidis from Stanford University stated (2005) that:

“.... research finding is less likely to be true when the studies conducted in a field are smaller; when effect sizes are smaller; when there are a greater number and lesser preselection of tested relationships; where there is greater flexibility in designs, definitions, outcomes, and analytical models; when there is greater financial and other interest and prejudice; and when more teams are involved in a scientific field in chase of statistical significance. Simulations show that for most study designs and settings, it is more likely for a research claim to be false than true".

A similar concern was expressed by Prof. Horton, the editor of "Lancet" medical journal (founded in 1823 a weekly peer-reviewed general medical journal which is among the world's oldest and best-known general medical journals) that stated (2015):

"...much of the scientific literature, perhaps half, may simply be untrue. Afflicted by studies with small sample sizes, little effects, invalid exploratory analyses, and flagrant conflicts of interest, together with an obsession for pursuing fashionable trends of dubious importance, science has taken a turn towards darkness." (p. 1380).

Thus, Evidence-Based practice as it is practiced today is not good enough since the pieces of evidence are not reliable and in too many instances became unsound "alibi" to justify malpractice. Perhaps the balance should tilt a bit towards more clinical input and maybe more room for clinician Masters that lost their status due to the pull of the evidence-based research approach to the extreme.

Despite these discouraging statements, it should be emphasized that these facts and opinions should not discourage practitioners from studying and examining scientific research, but they should be done carefully and cautiously.

\section{Evidence-Based Practice and the Polygraph}

Polygraph testing as a mean for detecting deception, which nowadays celebrates its one hundred's anniversary (depends on pinpointing the "day of birth") (Amsel, 2020), has started as a big promise that the then modern science had made to society in pursuing law, order, and justice, based on modern knowledge. However, along with the objective instrument's technical development, the moves in the testing procedure and the analysis of the physiological responses were based only on 
pseudo-objective unproven ideas and intuitions of a few outstanding individuals with fertile minds that most of them were not scientists or even had any scientific education. During the first fifty years, there was very little research activity that was conducted either in-house by polygraph examiners, most of it did not match basic scientific standards, or in the academia, typically unrelated to field practice (Orne, Thackray \& Paskewitz 1972). These two routes of polygraph-related activity lived side by side, hardly communicating until around 1970 when the scientific approach was boosted by Dr. David Raskin ${ }^{7}$ from Utah University, who started to research the polygraph techniques used in the field. His long-lasting dedication to uncompromised scientific research and development with his graduate students have resulted in field applications in the form of more valid examination techniques named after the university, the Utah techniques. In fact, that was a clear example of implementing the philosophy of Evidence-Based-Practice in the polygraph field without using this term. The group of scientific-oriented polygraph examiners and researchers he raised cast a giant positive impact on the field. It was not easy; they had to pave their way in a hostile environment comprised of conservative academic scientists on the one hand and many field practitioners who were engaged in a convenient non - scientifically based practice on the other. It took about twenty years to get accepted by enough core figures from both sides. At the same time, additional events in the polygraph field took place that collectively might have had a "game-changer" effect. The Office of Technology Assessment of the U.S. Congress (OTA) published in 1983 a report that criticized the polygraph validity and questioned, in particular, the use of the polygraph for pre-employment screening. Not without connection, a few years later, in 1988, the Employee Polygraph Protection Act (EPPA) was enacted in the U.S., banning the use of the polygraph for pre-employment and

\footnotetext{
${ }^{7}$ Raskin C.D. - A leading academic psychophysiologist from Utah University made a decision in the early 70 s to change his position from an Ivory sitting couch expert to an expert who are willing to check in person the existing field polygraph technique and open a program for graduate student to investigate the polygraph. During the years he raised several students who managed to enter Government polygraph units and brought a sense of scientific approach to the federal institutes and trough that to the private polygraph arena. Among them, his first student Dr. Barland who in the 80 s headed a research unit next to the federal polygraph school (DODPI, later became DACA and NCCA), Dr. Podlesny who has established a polygraph research unit in the FBI, Dr. Kircher that focused on developing the first commercial U.S. made computerized polygraph and an algorithm for analysis. Dr. Honts, Dr, Horowitz, who following years of serving as polygraph examiners and researcher in the public government sector have move to the academy. Dr Raskin and his students have developed the first polygraph technique that was based on scientific research, known as the Utah polygraph techniques.
} 
employment in most non-governmental businesses and abolished almost $90 \%$ of the private polygraph sector. Around that time, the Department of Defense Polygraph Institute (DODPI) included an in-house research unit that took the lead in the Polygraph instruction, research, and development. Following the EPPA 1988 Act, the U.S. Department of Energy asked the National Academy of Science to conduct a scientific review of the research on polygraph examinations that pertain to their validity and reliability, particularly for personnel security screening. In 2003 after a prolonged analysis of research and interviewing experts, the Academy published its conclusion (National Research Council, 2003) that included the following two assertions:

"Notwithstanding the limitations of the quality of the empirical research and the limited ability to generalize to real-world settings, we conclude that in populations of examinees such as those represented in the polygraph research literature, untrained in countermeasures, specific-incident polygraph tests can discriminate lying from truth-telling at rates well above chance, though well below perfection". (p. 4)

and

"A substantial portion of our recommended expanded research program should be administered by an organization or organizations with no operational responsibility for detecting deception and no institutional commitment to using or training practitioners of a particular technique. The research program should follow accepted standards for scientific research, use rules and procedures designed to eliminate biases that might influence the findings, and operate under normal rules of scientific freedom and openness to the extent possible while protecting national security”. (p. 9).

These events brought about an increase in the efforts to become a more scientifically based profession.

Scientific research, outside the context of Evidence-Based-Practice philosophy, was evident sporadically before Dr. Raskin started his project (e.g., Lykken, 1959, 1960; Gustafson \& Orne, 1963, 1964) and mostly dealt with the Guilty Knowledge Test that was the favorite paradigm in Lab research, but seldom in use in the field.

The move from field practice per-se to a more scientific oriented profession has started a bit earlier, in the late '60s, at the Israeli National Police with the involvement of a number of academic researchers from the Department of Psychology of the Hebrew University in Jerusalem, Dr. Kugelmass, Dr. Lieblich and later on Dr. BenShakhar (e.g., Kugelmass, et al., 1968; Ben-Shakhar et al.1970). The Israeli National Police Polygraph Unit became the Scientific Interrogation Laboratories, a section 
within the Criminal Identification and Forensic Science Division of Israel's National Police, and in years to come, during the 70 s, ' 80 s, and ' 90 s, lean towards scientific approach was more and more evident in particular with the work of in-house scientists Dr. Elaad, (e.g., Elaad, 1985, Elaad \& Schacher, 1985,) Dr. Ginton (e.g., Ginton et al., 1982, Ginton, 1985) and Mr. Kleiner (e.g., Elaad \& Kliener,1986; Kleiner, 2002). It is interesting to mention that during these years, the perception that polygraph use relates to applied psychology brought about a formal demand of having psychological education as a precondition for entering the polygraph unit of the Israeli police. A similar approach could be found at the time in Japan, but not in the U.S.

Over the years, the number of test formats and versions has grown to a full house of variations driven by intuitions or business interests with no real research support or objective justifications. The field became chaotic with no set of valid standards to compare to. The situation worsened with the dramatic worldwide growth of polygraph usage with thousands of examiners and millions of examinees per year. This undesirable situation led the American Polygraph Association, the world's biggest and most important polygraph body, to develop best practice principles and establish test standards. Given the then (and still) current bone-ton in the applied diagnostic and treatment world, it seemed the right step to adopt the Evidence-Based practice approach as the tool for improving the situation. That was also the way to improve the chances to get recognition by the Academia, which most opposed the use of the polygraph for field applications and in particular against the claim that the most common method - the CQT- is a valid scientific-based test.

If one follows Sackett et al. (1996) original definition of evidence-based practice to the dot, then polygraph practice does not meet that definition. Nowadays, polygraph practice strives to be as much standardized practice as possible, i.e., a checklist-type protocol. This type of practice actually contradicts Sackett et al. (1996) requirement to avoid any "cookbook" type practice and rely on the practitioner's discretion on a case-to-case basis. On the other hand, Mullen (2002) claim that the term evidence-based practice is used with two different meanings: Sackett et al.'s (1996) original definition of evidence-based practice, i.e., practitioners decision-making method, and an additional one associated with "best-practice" which defined by the Merriam-Webster Dictionary ${ }^{8}$ as: "a procedure that has been shown by research and experience to produce optimal results and that is established

\footnotetext{
${ }^{8}$ https://www.merriam-webster.com/dictionary/best\%20practice
} 
or proposed as a standard suitable for widespread adoption". Therefore, if we follow Mullen's (2002) definition, it will be more accurate to label the polygraph practice as an Evidence-Based Best Polygraph Practice rather than Evidence-Based Polygraph Practice. However, regardless of the different definitions' semantics, the heart and soul of these allegedly two different practical implications are the same: a practice that relies, based, and rooted on evidence attained by research.

The evidence-based practice philosophy resulted in a growing number of researches that supported the validity of some aspects of the polygraph practice. The top product was the various test data analysis (TDA), especially the OSS (Nelson, Handler \& Krapohl, 2007; Nelson, Krapohl \& Handler, 2008) and ESS (Nelson et al., 2011), which resulted in a more objective and stable analysis of the psychophysiological outcomes. The TDA studies come along with various validated test formats as shown in the Meta-Analytic Survey report of the American Polygraph Association (2011) and its update (Nelson, 2015) and noticeable growth in applied psychophysiological research and instrumentation.

Based on that, when honest practitioners asked by their clients: "How accurate are your findings? How certain are you that he is guilty? I want to make sure before taking any precautions against him". Depending on the validated test format utilized, the answer will come around 90\%. But, does the test format and the test data analysis research validity rates can be projected to an individual examinee?

Apart from the problem of base rates, which is a major factor in answering this question, there is also a concern to what degree can one be assured that the specific examinee in the specific circumstances of the actual test resembles the common examinee prototype and the range of circumstances that comprised the database for estimating the validity of the test format and version that was used by the examiner this time?

Suppose the specific examinee had trouble concentrating on the pre-test, and suppose in the sample used for the validity research, only ten percent of the subjects had a similar problem, and their effect on the validity outcome was as per their relative portion in the sample, is it reasonable to lean on the validity value that was found to estimate the degree of our confidence in the results found in a subject that his inability to concentrate affect $100 \%$ of the test not only $10 \%$ as was in the validity research? The same applied to circumstantial variability. These limitations are inherent in the statistical paradigms used for assessing the validity and, of course, in the evidence-based approach if it relies only on research evidence and 
ignores anything that was not researched scientifically yet or cannot be researched in one of those paradigms ever due to inherent issues. It is, therefore, that the Evidence-Based-Practice model in medicine includes clinical practice considerations. Shouldn't we too adopt this approach rather than ignore any information that originated from outside the Evidence-Based circle?

In pointing out the importance of individual differences in practicing medicine, Hippocrates said:

"It is more important to know what sort of person has a disease than to know what sort of disease a person has".

Adapting this view to the polygraph, we should think that although the polygraph test aims to detect the "disease" a person has, namely, detection of deception, in fact, we are busy differentiating between deceptive and truthful examinees. In that sense, we are focusing on the temporal qualities (deceptive or truthful subject, not as traits) that the individual carries during the test and the differences between individuals. The single person, the individuum, is the heart of our work. Learning the individuals' differences, though, would not prevent the "disease"', i.e., the deception to occur, it will prevent the polygraph examiner's disease, namely the errors in our work, and improve the quality of our decisions.

The various validated evidentiary test formats claim to have around $90 \%$ detection rate and around $10 \%$ inconclusive rate. As valuable as this information is, it does not provide the intersections of available individual characteristics such as age, education level, gender, etc. with success or failure of the test to reach correct outcomes. Analysis of these characteristics as well as more sophisticated factors but still simple, such as examiner-examinee opposite genders or age difference, may point out potential influencing factors that bear an effect on the outcome, which in return will enable examiners to fine-tune the test and alter the test to the examinee in a tailor-made style rather than altering the examinee to the test in a one size fits all style, not to mention a higher level of differentiation between the examinees' psychological characteristics and circumstantial differences that might affect the conduct of the test and its outcome.

This notion that contradicts the existing trend in the field that adores strict standardization, and strives for uniformity in the way the test is conducted and analyzed to the point of banning any move which is not Evidence-Based, was introduced to the field by Dr. Ginton in 2013 under the construct "Adaptive Polygraphy" 
(Ginton, 2013), calling to drive modern polygraphy towards developing a scientifically-based approach that follows the motto of

\section{"Different Things to Different People and Different Situations".}

Hence, we believe that polygraph research should shift its' focus from providing valuable test procedure information to providing an in-depth analysis of the examinees' profiles. Elements such as education level, age, gender, language proficiency, ethnic origin, law obedience or outlaw, emotional or rational personality type, past experience as an interviewee, and alike might influence the examiner-examinee inter-personal dynamics and, in return on the test outcome. The same investment is needed in researching the effects of different circumstances on test outcomes. Evidence-Based practice is more than searching for evidence under the street lamp. It should be active in initiating research in the dark corners that are currently ignored, and polygraph examiners are even warned not to search there.

In addition, we should keep in mind that Evidence-Based has two sides. The first, the most known side, is demonstrating success by research; in other words, it is an Evidence-Based success (e.g., this specific method works well, and it is supported by research that shows it). The second side is something that people, including scientists and practitioners, tend to ignore: evidence-based failure (e.g., this specific method produces mistakes, as shown by research). To prevent misunderstanding, it is not that the Evidence-Based approach fails to work, rather it is the success of the Evidence-Based approach to detect failure in practice. It is an unfortunate fact that this second type of Evidence-Based matters is being neglected.

Take the relevant-irrelevant (RIR) screening test format as an example; Krapohl \& Rosales (2014), Nelson (2015), and Krapohl \& Goodson (2015), concluded that the decision accuracy of this format is substantially poorer than other validated screening formats yet, many agencies and examiners continue practicing a format that fails, in spite of existing evidence.

Proven errors or mistakes and the enormous amount of information, sometimes critical, hiding in it, are not researched enough. Although it is human to err, mistakes are regarded as failure or, in some instances, as a character flaw, compelling people to play it safe, follow mainstream footsteps, and cling without flexibility to rigid evidence-based standards behavior that eventually leads to a stagnant science.

Evidence-Based Practice is, by definition, a sort of learning from experience by adopting specific research methods and standards, but this is by no means the only 
way to learn from experience. Polygraph research must adopt the Incident Investigations Approach that seeks to explain why a procedure failed, learn the lesson and avoid repeating the mistake, as well as why a procedure was successful to replicate it in the future. Only a systematic analysis of failure and success will ensure growth and development.

\section{Absence of evidence is not evidence of absence}

Evidence-Based practice in polygraph testing tends to accept as valid only procedure and methods that have been supported by research. As logical as this approach is, its counter side is the unfortunate wrong logic that treats the absence of statistical evidence as evidence of the absence of the researched phenomenon. In fact, failure to demonstrate the statistical significance of success in using a certain procedure or testing method does not prove they are a fault procedure or testing method. The use of $\alpha$ being it $0.05,0.01$, or any other value is meant to indicate the accepted degree of risk to be mistaken in acting under the assumption that the examined phenomenon exists whereas, in fact, it does not exist in reality. By no means is it a proof of the phenomenon's existence, and the failure to reach statistical significance is not a proof of its absence.

\section{Polygraph practice - Evidence-Based Technique and Art}

Decades of prolonged criticism on the validity of the Comparison Question Test (CQT), as well as the various unsystematic of unstandardized test formats, led our industry to continuous efforts to prove otherwise. An extreme strive for rigid standardization in the name of science tends to ignore the complexity of the polygraph practice, and it is based in a way on a simplistic and limited concept of what science is. Let alone that there is more than just science in practicing polygraphy. Along with the efforts in laying scientific foundations to the practice, we should consider the "art" aspects involved, such as the art of interpersonal communication, the art of the examiner to improvise in light of unexpected situations, and others. The probable-lie comparison question phrasing is an excellent example. As mentioned by Krapohl and Shaw in their comprehensive book (2015), under the so-called "Goldilocks Principle, the probable-lie comparison question "...must not be 'too hot' nor 'too cold' but 'just right'... “( p. 68), which requires a more artistically rather than a technical approach to set the "right temperature". (see also Ginton, 2009, p. 
210; Ginton, 2019, pp. 190-192). We should adopt the scientific methods not only in favor of standardizing our profession but also to improve our understanding of the "art" quality found in our work rather than suppress it in the name of science and standardization.

Over standardization, in its extreme form, may affect creativity, open-mindedness, flexibility, and human touch, which are essential for further future developments. The significance of personal and situational differences should not be ignored and overlooked even when it seems to be at odds with the developed rigid standardizations. The "tailor-made" adaptive polygraphy approach in conducting examinations should not be abolished in the name of science, and replaced by the standardized "scientific" "one size fits all" mediocre practice which is based on central tendencies, ignoring individual differences among the examinees, irregular cases or situations that are off the main center, or in Thomas Jefferson words paraphrasing Plato's (Laws) $)^{9}$ idea: "There is nothing more unequal than the equal treatment of unequal people" or as demonstrated graphically in Angus Maguire drawing

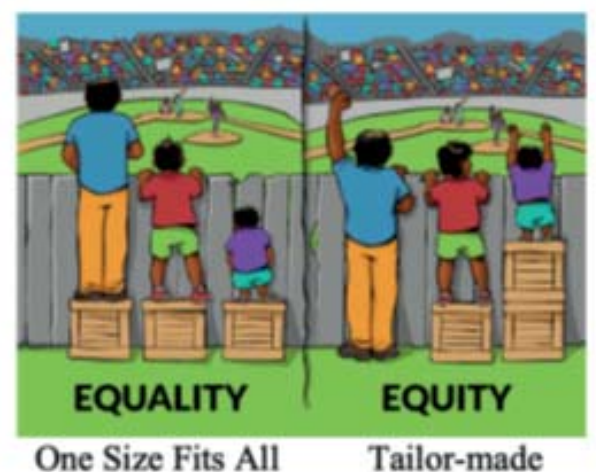

Reproduced with thanks to Interaction Institute for Social Change | Artist: Angus Maguire ${ }^{10}$.

"A picture is worth a thousand words" - Since we are discussing the merits and weaknesses of the Evidence-Based Practice approach, we should stress that one does not need research to see the evidence that one size fits all is sometimes problematic. But we do need research to verify it in polygraph practice to prove and improve the concept of the adaptive polygraph approach.

\footnotetext{
${ }^{9}$ Plato, Laws, Book 6, p. 757.

${ }^{10}$ https://interactioninstitute.org/illustrating-equality-vs-equity/
} 


\section{Epilogue}

As the polygraph profession followed the footsteps of medicine evidence-based practice and adopted its philosophy, we should follow the nowadays trend of medicine that shift from simple standardization of diagnoses and treatments to individualized, personalized medicine, which strives to tailor medical treatment to the individual characteristics of each patient considering the patient unique molecular, genetic profile and personal environmental factors recommending which medical treatments will be safe and effective for each patient, and which ones will not be. Personalized medicine can select a therapy or treatment protocol based on a patient's molecular profile that may not only minimize harmful side effects but ensure a more successful outcome (Olechno, 2016). Contrary to the existing trend in our field that, in a way, worship the strict standardization, we suggest steering modern polygraphy towards developing a scientifically-based approach that follows the motto of understanding and conducting "Different Things to Different People and Different Situations". In other words, we call for developing an adaptive approach or Adaptive Polygraphy (Ginton, 2013) that, based on acquired scientific knowledge, alters the test in a "tailor-made" mode to the individual examinee rather than alter the individual examinee to a "one size fits all" type test.

\section{References}

American Polygraph Association (2011), Meta-Analytic Survey of Criterion Accuracy of validated Polygraph Techniques. Polygraph, 40 (4), 194-305.

Amsel T., (2020), The Centennial Introspection Project 100 Years of Polygraph Practice. European Polygraph, 14 (1), 23-26.

Baker M., (2016), 1,500 scientists lift the lid on reproducibility. Nature, 533 (7604), 452-454.

Ben-Shakhar G., Lieblich I., \& Kugelmass S., (1970), Guilty knowledge technique: Application of signal detection measures. Journal of Applied Psychology, 54 (5), 409-413. https://doi.org/10.1037/h0029781

Bornmann L., Mutz R., (2015), Growth rates of modern science: A bibliometric analysis based on the number of publications and cited references. Journal of the Association for Information Science \& Technology, 66 (11), 2215-2222. 
Committee on Diagnostic Error in Health Care, (2015), Board on Health Care Services; Institute of Medicine; The National Academies of Sciences, Engineering, and Medicine; Balogh, E.P., Miller B.T., Ball J.R., (eds.) 2015.

Diagnostic Errors: Technical Series on Safer Primary Care, (2016), Geneva: World Health Organization. Licence: CC BY-NC-SA 3.0 IGO.

Elaad E., (1985), Decision Rules in Polygraph Examination in: IDENTA 85 - An International Conference held in Jerusalem Israel in 1985: Anti-terrorism, Forensic Science, Psychology in Police Investigations, 167-179, A Book of proceeding. First Published, 1985 Imprint Routledge. 2019. https://doi.org/10.4324/9780429036590.

Elaad E., and Kleiner, M., (1986), The stimulation test in polygraph field examinations: a case study.Journal Police Science \& Administration, 14 (4), 328-333.

Elaad E., and Schachar, E., (1985), Polygraph field validity. Polygraph, 14 (3), 217-223.

Fanelli D. (2009), How Many Scientists Fabricate and Falsify Research? A Systematic Review and Meta-Analysis of Survey Data. PLOS ONE 4(5) e5738. Bibcode:2009PLoSO...4.5738F. doi: 10.1371/journal.pone.0005738. PMC 2685008. PMID 19478950.

Ginton A., (1985), A Built-In Validity in Polygraph Field Examinations. in: IDENTA 85 - An International Conference held in Jerusalem Israel in 1985: Anti-terrorism, Forensic Science, Psychology in Police Investigations, 167-179, A Book of proceeding. First Published, 1985 Imprint Routledge. 2019. https://doi.org/10.4324/9780429036590.

Ginton A., (2009), Relevant Issue Gravity (RIG) Strength - A new concept in PDD that reframes the notion of Psychological Set and the role of attention in CQT polygraph examinations. Polygraph, 38 (3), 204-217

Ginton A., (2013, Sept), Adaptive Polygraph. [Paper Presentation]. The annual meeting of the American Polygraph Association, Orlando, FL. USA.

Ginton A., (2019), Essentials of the Relevant Issue Gravity (RIG) Strength; A Theoretical Framework for Understanding the Comparison Question Test (CQT) A detailed outline version. European Polygraph, 13 (4), 181-201. DOI: 10.2478/ep-2019-0013.

Ginton A., Daie N., Elaad E., and Ben-Shakhar G., (1982), A method for evaluating the use of the polygraph in a real-life situation. Journal of Applied Psychology, 67 (2), 131.

Gustafson L.A., \& Orne M.T., (1963), Effects of heightened motivation on the detection of deception. Journal of Applied Psychology, 47 (6), 408-411, https://doi. org/10.1037/h0041899. 
Gustafson L.A., \& Orne M.T., (1964), The effects of task and method of stimulus presentation on the detection of deception. Journal of Applied Psychology, 48, 383-387.

Guyatt G., Cairns J., Churchill D., et al. (1992), Evidence-Based Medicine: A New Approach to Teaching the Practice of Medicine, JAMA, 268 (17): 2420-2425.

Horton R., (2015), Offline: What is medicine's 5 sigma? The Lancet, 385 (9976).

Ioannidis J.P.A., (2005), Why Most Published Research Findings Are False? PLOS Med, 2 (8): e124.

Kleiner M., (2002), Physiological detection of deception in psychological perspectives: A theoretical proposal. In M. Kleiner (Ed.), Handbook of polygraph testing (pp. 127182). Academic Press.

Krapohl D.J. \& Goodson W., (2015), Decision accuracy for the Relevant-Irrelevant screening test: Influence of an algorithm in human decision-making. European Polygraph 9 (4), 189-208.

Krapohl D.J. \& Rosales T., (2014), Decision accuracy for the Relevant-Irrelevant screening test: A partial replication. Polygraph, 43 (1), 20-29.

Krapohl D.J., and Shaw P.K., (2015), Fundamentals of Polygraph Practice, Academic Press.

Kugelmass S., Lieblich I., Ben-Ishai A., Opatowski A., \& Kaplan M., (1968), Experimental evaluation of galvanic skin response and blood pressure change indices during criminal interrogation. Journal of Criminal Law, Criminology \& Police Science, 59 (4), 632-635. https://doi.org/10.2307/1141863.

Larsen P.O., \& von Ins M., (2010), The rate of growth in scientific publication and the decline in coverage provided by Science Citation Index. Scientometrics (2010) 84, 575-603, DOI 10.1007/s11192-010-0202-z.

Langlois J.P., (2002), "Making a Diagnosis”. In: Mengel M.B., Holleman W. L., Fields S.A., (eds.) Fundamentals of Clinical Practice ( $2^{\text {nd }}$ ed.). Kluwer Academic/Plenum Publisher.

Leach M.J., (2006), Evidence-based practice: A framework for clinical practice and research design. International Journal of Nursing Practice. 12 (5): 248-251.

Lykken D.T., (1959), The GSR in the detection of guilt. Journal of Applied Psychology, 43, 285-388.

Lykken D.T., (1960), The validity of the guilty knowledge technique: The effects of faking. Journal of Applied Psychology, 44 (4), 258-262. https://doi.org/10.1037/ h0044413. 
Mullen E.J., (2002, July), Evidence-Based Knowledge: Designs for Enhancing Practitioner Use of Research Findings (a bottom-up approach). [Paper Presentation]. The 4th International Conference on Evaluation for Practice, University of Tampere, Tampere, Finland. http://www.uta.fi/laitokset/sospol/eval2002/EvidenceF2002.PDF.

National Academies of Science, Engineering, and Medicine. (2019), Reproducibility and Replicability in Science. Washington, DC: The National Academies Press. https:// doi.org/10.17226/25303.

National Research Council (2003), The Polygraph and Lie Detection. Committee to Review the Scientific Evidence on the Polygraph. Division of Behavioral and Social Sciences and Education. Washington, DC: The National Academies Press. https://doi. org/10.17226/10420.

Nelson R. (2015), Appendix B: 2015 Update to the American Polygraph Association 2011 meta-analytic survey of validated polygraph techniques. In D. Krapobl and P. Shaw Fundamentals of Polygraph Practice. Academic Press.

Nelson R., Handler M. \& Krapohl D. (2007, Sept), Development and validation of the Objective Scoring System, version 3. [Poster presentation]. The annual meeting of the American Polygraph Association, New Orleans, LA. USA.

Nelson R., Handler M., Shaw P., Gougler M., Blalock B., Russell C., Cushman B. \& Oelrich M., (2011), Using the Empirical Scoring System. Polygraph, 40, 67-78.

Nelson R., Krapohl D. \& Handler M., (2008), Brute force comparison: A Monte Carlo study of the Objective Scoring System version 3 (OSS-3) and human polygraph scorers. Polygraph, 37, 185-215.

Olechno J., (2016), Individualized medicine vs. precision medicine. DDNews, 12,5.

Open Science Collaboration, (2015), Estimating the reproducibility of psychological science. Science, 349 (6251).

Orne M.T., Thackray R.I. \& Paskewitz D.A., (1972), On the detection of deception, A model for the study of the physiological effects of psychological stimuli. In: N.S. Greenfield \& R.A. Sternbach (Eds.), Handbook of psychophysiology. Holt, Rinehart \& Winston, 1972, 743-785.

Pashler H., Wagenmakers E.J., (2012), Editors' Introduction to the Special Section on Replicability in Psychological Science: A Crisis of Confidence? Perspectives on Psychological Science. 7 (6). 528-530.

Peng R., (2015), The reproducibility crisis in science: A statistical counterattack, Significance, 12 (3), 30-32. 
Sackett D.L., Rosenberg W.C., Muir Gray J.A., Haynes R.B., Richardson W.S., (1996), Evidence based medicine: what it is and what it isn't. BMJ, 312, 71-72.

Straus S.E., Glasziou P., Richardson W.S. \& Haynes R.B. (2011), Evidence-based medicine: How to practice and teach EBM ( $4^{\text {th }}$ ed.), Churchill Livingstone.

The Office of Technology Assessment of the U.S. Congress -OTA- (1983), Scientific Validity of Polygraph Testing: A Research Review and Evaluation.,

Webb S., (2001), Some consideration on the validity of evidence-based practice in social work. British Journal of Social Work 31 (1), 57-79.

Youngstrom E.A., Choukas-Bradley S., Calhoun C.D., Jensen-Doss A., (2015), Clinical Guide to the Evidence-Based Assessment Approach to Diagnosis and Treatment. Cognitive and Behavioral Practice, 2 (1). 